\title{
Palliative Care Services at a Cancer Center in Janakpur Nepal
}

\author{
Sanjay Gupta, Anshu K Thakur, Reecha Mishra, Pamesh Jha \\ Binaytara Foundation Cancer Center, Kadam Chowk, Kapileshwore Marg, Janakpurdham, Nepal
}

\section{Corresponding author:}

\section{Sanjay Gupta, MBBS, MD}

Binaytara Foundation Cancer Center, Kadam Chowk, Kapileshwore Marg, Janakpurdham, Nepal Email:sanjaygupta@binayfoundation.org

\begin{abstract}
Introduction

Palliative care is one of the essential components of cancer care in Nepal. Most of our cancer patients present to the hospital quite late. In our everyday practice, we encounter many progressive incurable cancer patients who need palliative care. The goal of the palliative care is to relieve the pertinent symptoms or to improve quality of life (QoL) for patients with advanced cancer. We reviewed the palliative care services to the cancer patients at our center.
\end{abstract}

\section{Methods}

Retrospective analysis of the cancer patients who received palliative care alone or in adjunct to curative treatment in Binayatara Cancer Center since its establishment in December 2018 was done.

\section{Results}

A total of 108 patients received palliative care of which most were at advanced stage of disease (Stage IV). The male to female ratio was $1: 1.45$. The most common complaints for their visit was pain (62\%) followed by nause and vomiting (32\%), constipation (28\%), anorexia (22\%), shortness of breath, abdominal distension, seizure. Most of the patients with pain needed morphine $(65 \%)$ for pain management followed by tramadol (25\%) and NSAIDs (10\%). Few of them were also supplemented with TCAs and anticonvulsants. Abdominal distension due to ascites was managed by ascitic tapping, Albumin supplementation while shortness of breath due to pleural effusion was managed by pleural fluid tapping. In one patient with pleural effusion, chest tube was inserted. Three patients with seizure due to brain metastasis were managed with anticonvulsant. A patient of prostate cancer with bony metastasis was managed with surgical orchidectomy.

\section{Conclusion}

While considering the appropriate and effective use of palliative care, a palliative care physician is often confronted with a full range of multidisciplinary treatment options, and technical considerations that could potentially relieve some symptoms of advanced cancer in our settings.

Keywords: Advanced cancer, palliative care, quality of life 